\title{
Diastereoselective Aldol Addition Reactions of a Chiral Methyl Ketone Trichlorosilyl Enolate under Lewis Base Catalysis
}

Scott E. Denmark,* Shinji Fujimori

Roger Adams Laboratory, Department of Chemistry, University of Illinois, Urbana, IL 61801, USA

Fax (217) 333-3984; E-mail: sdenmark@uiuc.edu

Received 7 February 2001

\begin{abstract}
The trichlorosilyl enolate of a chiral methyl ketone bearing an oxygen substituent (OTBS) on the $\beta$-position undergoes highly diastereoselective aldol addition to a variety of achiral aldehydes in good yield. The stereochemical course of the reaction is primarily controlled by the configuration of the chiral phosphoramide which serves as an effective catalyst for this transformation.
\end{abstract}

Key words: aldol addition, trichlorosilyl enolate, Lewis base catalysis, diastereoselection

The stereocontrolled aldol addition reaction remains one of the most powerful and versatile carbon-carbon bond forming reactions in organic synthesis. ${ }^{1}$ Among the manifold variants of this venerable process, the addition of unsubstituted (i.e. methyl ketone) enolates has presented a particular challenge. ${ }^{2}$ Although the reasons for this behavior are still under investigation, one popular rationalization is the availability of competing diastereomeric transition structures (twist-boats) resulting from the reduced steric demand of the nucleophile. ${ }^{3}$

As part of our exploration of the chemistry of enoxytrichlorosilanes, ${ }^{4 a}$ we have recently described a broad investigation of the catalytic enantioselective addition of methyl ketone trichlorosilyl enolates, Scheme 1. ${ }^{4 \mathrm{~b}}$ These reactive enolates can be generated in situ by simple mercuric acetate catalyzed exchange of the TMS enol ethers with silicon tetrachloride. Subsequent aldol reactions are catalyzed by chiral phosphoramides to afford the addition products in excellent yield and good enantioselectivities (3.2-24.0/1 er).

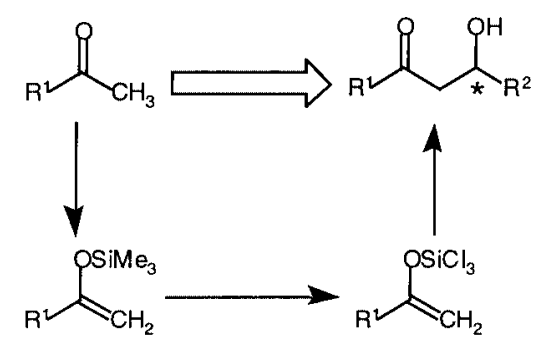

Scheme 1

To expand the synthetic utility of these enolates, we also studied the addition reactions of chiral methyl ketone enolates bearing an $\alpha$ oxygen substituent, e.g. ii, Scheme 2. This enolate (also readily generated in situ from the TMS enol ether i) undergoes addition in the matched case with phosphoramide $(R, R)$-1a (Chart 1) to generate the aldol product iii with high 1,4-syn diastereoselection. However, it was not possible to reverse the sense of diastereoselection by the use of $(S, S)$-1a.

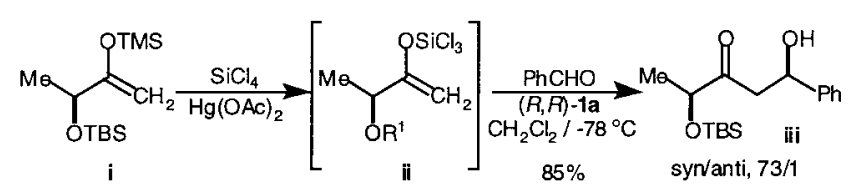

Scheme 2

In continuation of our generalization of this process, we have now extended the scope of chiral methyl ketone structures to include those bearing an oxygen substituent at the $\beta$-position, 2, Figure 1. Whereas structures of this type do not give highly diastereoselective aldol additions as their lithium enolates, ${ }^{5}$ Paterson has shown that the dicyclohexylboron enolates can give good 1,4-syn diastereoselection. ${ }^{6}$ Interestingly, the use of the (-)-IPC ligand significantly improves selectivity, but the (+)-IPC ligand gives the same selectivity as the achiral group. We describe herein the in-situ generation and aldol reaction of the trichlorosilyl enolate $\mathbf{4}$ derived from $\mathbf{2}$ and the ability of chiral phosphoramides to control the diastereoselectivity of the addition.

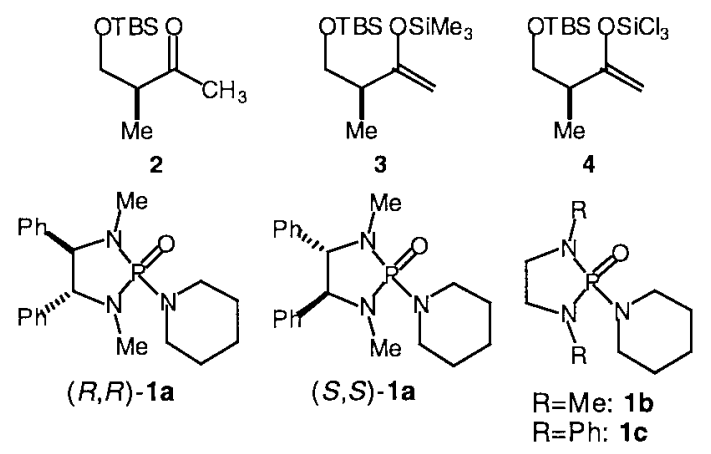

Figure 1

The methyl ketone $\mathbf{2}$ was easily prepared in three steps from enantiopure $(S)-(+)$-methyl $\beta$-hydroxyisobutyrate, Scheme 3. Conversion of the methyl ester to the Weinreb amide $\mathbf{5}^{7}$ proceeded in $88 \%$ yield with the agency of trimethylaluminum. Following protection of $\mathbf{5}$ as a tert-bu- 
tyldimethylsilyl ether, $\mathbf{6}$, the amide was converted to the methyl ketone 2 by controlled addition of methylmagnesium bromide in $91 \%$ yield. Regioselective formation of the unsubstituted trimethylsilyl enol ether $\mathbf{3}$ was effected by the combination of LDA and TMSCl at $-78^{\circ} \mathrm{C}$.

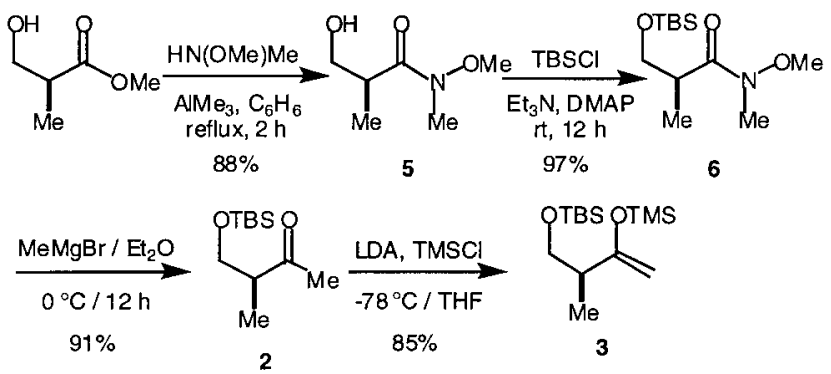

Scheme 3

For initial studies, the trichlorosilyl enolate $\mathbf{4}$ was prepared in bulk and purified by distillation. By following our established procedure ${ }^{8}\left(1 \mathrm{~mol} \% \mathrm{Hg}(\mathrm{OAc})_{2}, 2.0\right.$ equiv $\mathrm{SiCl}_{4}$ ) the metathesis of $\mathbf{3}$ to $\mathbf{4}$ proceeded readily, but the distilled product was contaminated with residual $\mathrm{Hg}(\mathrm{OAc})_{2}$. This problem was circumvented by the use of $\mathrm{Pd}(\mathrm{OAc})_{2}(5 \mathrm{~mol} \%)$ which we have found also to be an effective catalyst for this transformation. ${ }^{9}$

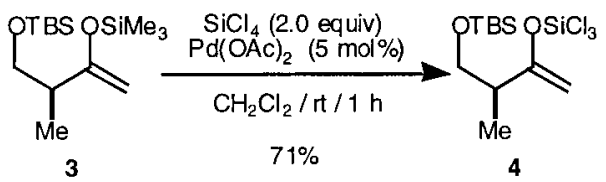

Scheme 4

Optimization of the aldol addition reactions of $\mathbf{4}$ with benzaldehyde involved a survey of the reaction concentration, catalyst loading and structure. The results are collected in Table 1. The chiral phosphoramide $(R, R)-\mathbf{1 a}$ could effectively promote the addition with as little as $5 \mathrm{~mol} \%$ catalyst at a $0.5 \mathrm{M}$ reaction concentration. The configurational assignment of the major diastereomer as syn-7 was secured by single crystal X-ray analysis of the diol obtained by deprotection with PPTS in EtOH. Interestingly, the reaction selectivity was not dependent on the loading of the catalyst (cf. entries 2-4). The enantiomeric catalyst $(S, S)$-1a, was also effective in promoting the addition with as little as $5 \mathrm{~mol} \%$ at $0.5 \mathrm{M}$ concentration. Lower reaction concentrations can be used if the catalyst loading is increased. Although the selectivities are not as high as from $(R, R)-\mathbf{1 a}$, we were pleased that the catalyst was able to reverse stereoselectivity seen with $(R, R)$-1a and produce the anti-7 as the major product. To establish the intrinsic, internal diastereoselectivity ${ }^{10}$ in this catalyzed process the achiral phosphoramides $\mathbf{1 b}$ and $\mathbf{1 c}$ were tested. The methyl derivative $\mathbf{1 b}$ showed as similar reactivity as $(R, R)-\mathbf{1 a}$ and produced syn-7 with modest selectivity as the major isomer.
Table 1 Optimization of Aldol Additions of $\mathbf{4}$ with Phosphoramides 1a, 1b, 1c.

\begin{tabular}{ccccccc}
\hline entry & catalyst & conc., $\mathrm{M}$ & $\begin{array}{c}\text { loading, } \\
\text { mol \% }\end{array}$ & $\begin{array}{c}\text { time, } \\
\mathrm{h}\end{array}$ & $\begin{array}{c}\text { yield, } \\
\%^{a}\end{array}$ & $\begin{array}{c}\text { syn/anti } \\
7^{b}\end{array}$ \\
\hline 1 & $(R, R)-\mathbf{1 a}$ & 0.1 & 5 & 2 & $43^{c}$ & $14 / 1$ \\
2 & $(R, R)-\mathbf{1 a}$ & 0.5 & 5 & 2 & 79 & $10 / 1$ \\
3 & $(R, R)-\mathbf{1 a}$ & 0.5 & 10 & 1 & 73 & $10 / 1$ \\
4 & $(R, R)-\mathbf{1 a}$ & 0.5 & 15 & 1 & 71 & $10 / 1$ \\
5 & $(S, S)-\mathbf{1 a}$ & 0.1 & 15 & 3 & $57^{c}$ & $1 / 9$ \\
6 & $(S, S)-\mathbf{1 a}$ & 0.2 & 10 & 2 & 81 & $1 / 6$ \\
7 & $(S, S)-1 \mathbf{a}$ & 0.2 & 15 & 2 & 72 & $1 / 6$ \\
8 & $(S, S)-1 \mathbf{a}$ & 0.5 & 5 & 2 & 76 & $1 / 6$ \\
9 & $\mathbf{1 b}$ & 0.1 & 5 & 5 & $51^{c}$ & $5 / 1$ \\
10 & $1 \mathbf{c}$ & 0.1 & 15 & 12 & $31^{c}$ & $2 / 1$ \\
\hline
\end{tabular}

${ }^{a}$ yield of chromatographically homogeneous material. ${ }^{b}$ determined by supercritical fluid chromatography (Chiralpak ${ }^{\circledR}$ OD; 175 bar, 2.5 $\mathrm{mL} / \mathrm{min}, 1.7 \% \mathrm{MeOH}, \lambda=220 \mathrm{~nm}) .{ }^{c}$ incomplete reaction.

The rate, yield and selectivity of the aldol reactions of $\mathbf{3}$ were sufficiently interesting to examine the generality of the process with other aromatic, olefinic, acetylenic and aliphatic aldehydes possessing various substitution patterns (Figure 2). However, to further illustrate the synthetic utility of these reagents, we performed all of these additions with in-situ-generated $\mathbf{4}$. For this purpose, we returned to the use of $\mathrm{Hg}(\mathrm{OAc})_{2}$ because lower loadings were needed to accomplish the metathesis compared to $\mathrm{Pd}(\mathrm{OAc})_{2}(1 \mathrm{~mol} \%$ vs. $5 \mathrm{~mol} \%)$ and we wished to avoid complications arising from phosphoramide poisoning by the metal salt. To assure complete reaction with a variety of aldehydes, we chose to use $10 \mathrm{~mol} \%$ catalyst at $0.5 \mathrm{M}$ concentration as the standard condition and simply varied reaction time. The results of this study are collected in Table 2. ${ }^{11}$

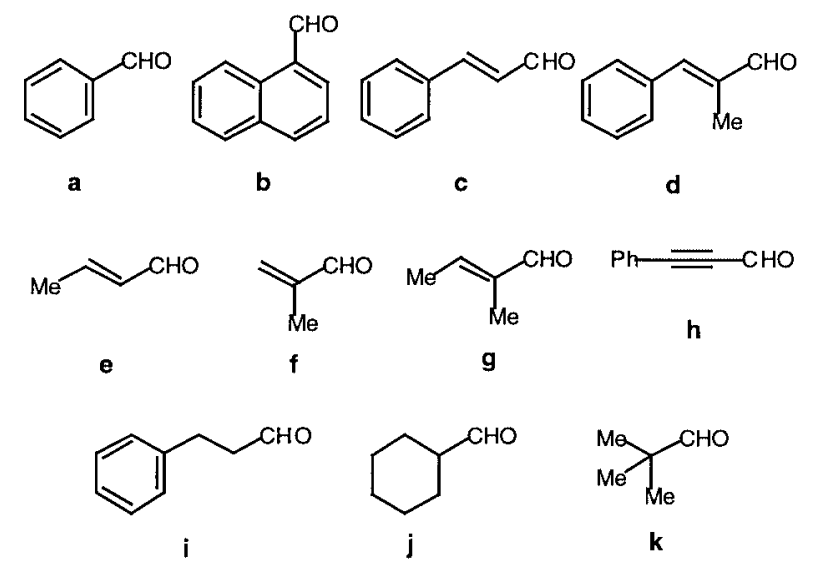

Figure 2

The first entry in Table 2 shows clearly that the in-situ procedure is not only operationally simpler, but also leads to superior results. The overall yield for reaction with benzaldehyde is higher and the selectivities for both 
Table 2 Survey of Aldehyde Structure in the Aldol Additions of In-situ Generated $\mathbf{4}$ with $(R, R)-\mathbf{1 a},(S, S)$-1a, and $\mathbf{1 b}$.

\begin{tabular}{ccccc}
\multicolumn{3}{c}{$c$} \\
\hline
\end{tabular}

${ }^{a}$ Yield of analytically pure material. ${ }^{b}$ Determined by supercritical fluid chromatography (Chiralpak ${ }^{\circledR}$ OD; 150 bar, $3.0 \mathrm{~mL} / \mathrm{min}$, $\lambda=220 \mathrm{~nm}$ ). ${ }^{c}$ Determined by supercritical fluid chromatography on the benzoate. ${ }^{d}$ incomplete reaction. ${ }^{e}$ Determined by ${ }^{1} \mathrm{H}$ NMR analysis in benzene- $d_{6} .{ }^{f} 10 \mathrm{~mol} \% \mathbf{1 b}$ used as catalyst.

catalysts is markedly improved. Similarly 1-naphthaldehyde gives high yields and good selectivities in both directions. The olefinic aldehydes (entries 3-7) all give good yields, but selectivities are quite variable and generally much better for the $(R, R)$ catalyst. The beneficial effect of an $\alpha$-substituent is seen by comparison of entries 3 and 4 as well as 5 and 7 . The high selectivity for methacrolein itself (entry 6) further supports the importance of branching near the carbonyl group. Not surprisingly then, the acetylenic aldehyde is the least selective of the cases (entry 8). In the aliphatic series, the yields were significantly lower and in entries 9 and 10 the reactions were still not complete after 10 h. ${ }^{12}$ Nonetheless, the selectivities follow the same trend, increasing with increasing $\alpha$-branching. The internal selectivity was also established under these conditions by reaction of $\mathbf{3}$ with benzaldehyde under catalysis by $\mathbf{1 b}$ (entry 12). The preference for formation of the syn isomer was seen again at the same level compared to entry 9 , Table 1 .

With the exception of the unhindered aliphatic aldehydes, the yields for all these reactions are synthetically useful. Moreover, the selectivities for production of the syn isomer make this a viable method for acyclic stereocontrol competitive with the best auxiliary-based methods. ${ }^{6}$ In that context, it is interesting to note that, despite the modest selectivities, we were able to reverse the intrinsic internal preference with a chiral catalyst, clearly a result of dominant external control. ${ }^{10}$ This stands in contrast to the persistence of internal control with chirally modified boron enolates. The ability of a non-covalently attached and conformationally ill-defined additive to influence the stereochemical course of a reaction in a way that large, covalently attached modifiers cannot, serves to illustrate the power of asymmetric catalysis and to provide an important lesson for understanding the origin of stereocontrol in these fundamental processes.

Extension of these studies to chiral ethyl ketones as well as chiral ketones with more remote stereocenters is in progress.

\section{Preparation of (+)-(S)- $N$-Methoxy- $N$-methyl-3-tert-butyldime-} thyl-silyloxy-2-methyl-propionamide (6)

To a solution of the hydroxy amide $\mathbf{5}(5.84 \mathrm{~g}, 39.7 \mathrm{mmol})$ in $120 \mathrm{~mL}$ of THF were added tert-butyldimethylchlorosilane $(7.78 \mathrm{~g}, 51.6$ mmol, 1.3 equiv), 4-dimethylaminopyridine $(485 \mathrm{mg}, 3.97 \mathrm{mmol}$, 0.1 equiv) and triethylamine ( $13.8 \mathrm{~mL}, 99.3 \mathrm{mmol}, 2.5$ equiv). The reaction mixture was stirred at r.t. for $12 \mathrm{~h}$. The solvent was removed under reduced pressure and the residue was triturated in 100 $\mathrm{mL}$ of ether. The insoluble material was removed by filtration. The filtrate was washed sequentially with $10 \mathrm{~mL}$ of $15 \% \mathrm{v} / \mathrm{v}$ aqueous acetic acid, $10 \mathrm{~mL}$ of water and $10 \mathrm{~mL}$ of sat. aq. $\mathrm{NaHCO}_{3}$. The ether solution was dried $\left(\mathrm{MgSO}_{4}\right)$ and was concentrated to give 12.3 $\mathrm{g}$ of crude material. The crude product was distilled to afford the silyloxy amide $6(10.0 \mathrm{~g}, 97 \%)$ as a colorless oil. Data for $\mathbf{6}$ : bp: $103{ }^{\circ} \mathrm{C} / 0.6 \mathrm{mmHg},{ }^{1} \mathrm{H}$ NMR: $\left(500 \mathrm{MHz}, \mathrm{CDCl}_{3}\right) 3.82$ - 3.85, 3.51 3.54 (ABX, $2 \mathrm{H}) ; 3.71(\mathrm{~s}, 3 \mathrm{H}) ; 3.19(\mathrm{~s}, 3 \mathrm{H}) ; 3.13-3.22(\mathrm{~m}, 1 \mathrm{H})$; $1.07(\mathrm{~d}, J=6.9,3 \mathrm{H}) ; 0.87(\mathrm{~s}, 9 \mathrm{H}) ; 0.04(\mathrm{~d}, J=4.9,6 \mathrm{H}),{ }^{13} \mathrm{C} \mathrm{NMR}$ : $\left(125 \mathrm{MHz}, \mathrm{CDCl}_{3}\right) 176.29 ; 65.90 ; 61.68 ; 38.23 ; 31.17 ; 26.03$; 18.43; 13.95; -5.29, -5.31, IR: (neat) 1666 (s), MS: (FI) $261\left(\mathrm{M}^{+}\right.$, $0.57),[\alpha]_{\mathrm{D}}{ }^{24}+24.4^{\circ}\left(\mathrm{CHCl}_{3}, \mathrm{c}=1.0\right)$ Anal. Calc for $\mathrm{C}_{12} \mathrm{H}_{27} \mathrm{NO}_{3} \mathrm{Si}$ (261.43), Calc. C 55.13\%; H 10.41\%; N 5.36\%, Found: C 55.05\%; H $10.16 \%$; N $5.35 \%$

Preparation of (+)-(S)-4-tert-Butyldimethylsilyloxy-3-methyl-2butanone (2)

The amide 6 ( $2.70 \mathrm{~g}, 10.3 \mathrm{mmol})$ was dissolved in $30 \mathrm{~mL}$ of diethyl ether at $0{ }^{\circ} \mathrm{C}$. To this solution was slowly added $3.0 \mathrm{M}$ solution of $\mathrm{MeMgBr}$ in ether $(4.48 \mathrm{~mL}, 13.4 \mathrm{mmol}, 1.3$ equiv). The reaction mixture was stirred at $0{ }^{\circ} \mathrm{C}$ for $1 \mathrm{~h}$, then was gradually warmed up to r.t. over 11 hours. The reaction mixture was quenched by slow addition of sat. aq. $\mathrm{NH}_{4} \mathrm{Cl}$ (ca. $30 \mathrm{~mL}$ ). The layers were separated and the aqueous layer was extracted twice with $50 \mathrm{~mL}$ of ether. The extracts were combined, dried $\left(\mathrm{MgSO}_{4}\right)$ and concentrated to give $2.48 \mathrm{~g}$ of crude oil. The crude product was purified by chromatography (pentane/ether, $3 / 1, \mathrm{SiO}_{2}$ ) followed by distillation to afford $\mathbf{2}$ $(2.02,91 \%)$ as a colorless liquid. Data for 2 : bp: $55^{\circ} \mathrm{C} / 1.0 \mathrm{mmHg}$, ${ }^{1} \mathrm{H}$ NMR: (500 MHz, $\left.\mathrm{CDCl}_{3}\right) 3.63$ - 3.74 (ABX, 2H); 2.74 (sext, $J=7.1,1 \mathrm{H}) ; 2.18(\mathrm{~s}, 3 \mathrm{H}) ; 1.07(\mathrm{~d}, J=6.8,3 \mathrm{H}) ; 0.87$ (s, 9H); 0.03 $(\mathrm{d}, J=4.4,6 \mathrm{H}),{ }^{13} \mathrm{C}$ NMR: $\left(125 \mathrm{MHz}, \mathrm{CDCl}_{3}\right)$ 212.24; 65.65; 49.53; 29.78; 26.02; 18.40; 13.13; -5.29, -5.31, IR: (neat) 1718 (s), MS: (FI) $217\left(\mathrm{M}^{+}, 2.51\right),[\alpha]_{\mathrm{D}}{ }^{24}+33.5^{\circ}\left(\mathrm{CHCl}_{3}, \mathrm{c}=1.0\right)$ Anal. Calc for $\mathrm{C}_{11} \mathrm{H}_{24} \mathrm{O}_{2} \mathrm{Si}$ (216.39), Calc. C 61.05\%; $\mathrm{H} \mathrm{11.18 \% ,} \mathrm{Found:} \mathrm{C}$ $60.99 \%$; $11.29 \%$

\section{Preparation of (S)-4-tert-Butyldimethylsilyloxy-3-methyl-2-tri-} methylsilyloxy-1-butene (3)

$n$-Butyllithium (1.54 $\mathrm{M}$ in hexane, $11.1 \mathrm{~mL}, 7.18 \mathrm{mmol}, 1.4$ equiv) was added dropwise to a solution of diisopropylamine $(1.67 \mathrm{~mL}$, $11.9 \mathrm{mmol}, 1.5$ equiv) in $30 \mathrm{~mL}$ of THF at $0^{\circ} \mathrm{C}$. The reaction mixture was stirred at $0{ }^{\circ} \mathrm{C}$ for $10 \mathrm{~min}$ then was cooled to $-78^{\circ} \mathrm{C}$. To the reaction mixture were added TMSCl $(1.51 \mathrm{~mL}, 11.9 \mathrm{mmol}, 1.5$ equiv) and the ketone $2(1.71 \mathrm{~g}, 7.90 \mathrm{mmol})$ and the reaction mixture was stirred for $30 \mathrm{~min}$. The reaction mixture was gradually warmed up to $0{ }^{\circ} \mathrm{C}$ and was quenched with $40 \mathrm{~mL}$ of cold $\mathrm{H}_{2} \mathrm{O}$. The layers were separated and the aqueous layer was extracted twice 
with pentane. The combined extracts were washed with $50 \mathrm{~mL}$ of sat. aq. $\mathrm{CuSO}_{4}, 25 \mathrm{~mL}$ of water and $50 \mathrm{~mL}$ of brine. The organic extracts were dried $\left(\mathrm{Na}_{2} \mathrm{SO}_{4}\right)$ and were concentrated to give $2.32 \mathrm{~g}$ of crude oil. After distillation the enolate $3(1.94,85 \%)$ was obtained as colorless oil. Data for 3: bp: $64{ }^{\circ} \mathrm{C} / 1.0 \mathrm{mmHg},{ }^{1} \mathrm{H}$ NMR: $(500$ $\left.\mathrm{MHz}, \mathrm{CDCl}_{3}\right) 4.05(\mathrm{~d}, J=0.7,1 \mathrm{H}) ; 4.03(\mathrm{~d}, J=1.0,1 \mathrm{H}) ; 3.36-3.70$ $(\mathrm{ABX}, 2 \mathrm{H}) ; 2.27$ (sext, $J=6.8,1 \mathrm{H}) ; 1.01(\mathrm{~d}, J=6.8,3 \mathrm{H}) ; 0.89$ (s, 9H); 0.20 (s, 9H); $0.04(\mathrm{~s}, 6 \mathrm{H}),{ }^{13} \mathrm{C}$ NMR: $\left(125 \mathrm{MHz}, \mathrm{CDCl}_{3}\right)$ $160.75 ; 89.39 ; 66.02 ; 43.14 ; 25.9218 .34 ; 14.99 ; 0.13 ;-5.37$, IR: (neat) $1657(\mathrm{w}) ; 1626(\mathrm{~m}), \mathrm{MS}$ : (FI) $289\left(\mathrm{M}^{+}, 100\right)$, Anal. Calc for $\mathrm{C}_{14} \mathrm{H}_{32} \mathrm{O}_{2} \mathrm{Si}_{2}$ (288.57), Calc. C $58.27 \%$; $\mathrm{H} 11.18 \%$, Found: C $58.05 \% ; \mathrm{H} 11.02 \%$

Preparation of $(S)$-4-tert-Butyldimethylsilyloxy-3-methyl-2-trichlorosilyloxy-1-butene (4)

Silicon tetrachloride ( $1.74 \mathrm{~mL}, 15.1 \mathrm{mmol}, 2$ equiv) was added to a solution of $\mathrm{Pd}(\mathrm{OAc})_{2}(85 \mathrm{mg}, 0.38 \mathrm{mmol}, 0.05$ equiv) in $7.57 \mathrm{~mL}$ of $\mathrm{CH}_{2} \mathrm{Cl}_{2}$. The trimethylsilyl enol ether $3(2.18 \mathrm{~g}, 7.57 \mathrm{mmol})$ was added to the reaction mixture using a cannula, and the reaction mixture was stirred at r.t. for $1 \mathrm{~h}$. The trichlorosilyl enol ether $4(1.88 \mathrm{~g}$, $71 \%$ ) was obtained by fractional distillation of the reaction mixture as a clear liquid. Data for 4: bp: $70-72{ }^{\circ} \mathrm{C} / 0.1 \mathrm{mmHg},{ }^{1} \mathrm{H} \mathrm{NMR}$ : $\left(400 \mathrm{MHz}, \mathrm{CDCl}_{3}\right) 4.58(\mathrm{~d}, J=2.4,1 \mathrm{H}) ; 4.55(\mathrm{~d}, J=2.4,1 \mathrm{H}) ; 3.46$ $-3.68(\mathrm{ABX}, 2 \mathrm{H}) ; 2.39$ (sext, $J=6.6,1 \mathrm{H}) ; 1.07(\mathrm{~d}, J=6.8,3 \mathrm{H})$; 0.89 (s, 9H); $0.04(\mathrm{~s}, 6 \mathrm{H})$

General Procedure for the Aldol Reaction of $3:^{13}$ (2S,5R)-1-tertButyldimethylsilyloxy-2-methyl-5-hydroxy-5-phenyl-3-pentanone (syn-7a)

To a suspension of mercury(II) acetate $(3.2 \mathrm{mg}, 0.01 \mathrm{mmol}, 0.01$ equiv) in $1 \mathrm{~mL}$ of $\mathrm{CH}_{2} \mathrm{Cl}_{2}$ were added $\mathrm{SiCl}_{4}(0.23 \mathrm{~mL}, 2.0 \mathrm{mmol}, 2.0$ equiv) and the trimethylsilyl enol ether $3(288 \mathrm{mg}, 1.00 \mathrm{mmol})$. The reaction mixture was stirred at r.t. for $30 \mathrm{~min}$. The excess $\mathrm{SiCl}_{4}$ and the solvent were removed under vacuum. To the residue was added solution of $(R, R)-\mathbf{1 a}(36.9 \mathrm{mg}, 0.10 \mathrm{mmol}, 0.10$ equiv) in $2 \mathrm{~mL}$ of $\mathrm{CH}_{2} \mathrm{Cl}_{2}$ via a cannula. The reaction mixture was cooled to $-78^{\circ} \mathrm{C}$ before the addition of benzaldehyde $(102 \mu \mathrm{L}, 1.00 \mathrm{mmol}, 1$ equiv). The reaction mixture was stirred at $-78^{\circ} \mathrm{C}$ for $2.5 \mathrm{~h}$, and then was quenched by pouring into a vigorously stirring $5 \mathrm{~mL}$ of cold sat. aq. $\mathrm{NaHCO}_{3}$. The resulting slurry was stirred for $1 \mathrm{~h}$ and was filtered through Celite. The layers were separated and the aqueous layer was extracted with $20 \mathrm{~mL}$ of $\mathrm{CH}_{2} \mathrm{Cl}_{2}$. The combined organic extracts were washed with $5 \mathrm{~mL}$ of brine, dried $\left(\mathrm{Na}_{2} \mathrm{SO}_{4}\right)$ and concentrated. The crude product was chromatographed (hexane/ether, 5/1, $\mathrm{SiO}_{2}$ ) to give $7 \mathbf{a}(268 \mathrm{mg}, 0.83 \mathrm{mmol}, 83 \%)$ as viscous colorless oil. Data for syn-7a: ${ }^{1} \mathrm{H}$ NMR: $\left(500 \mathrm{MHz}, \mathrm{CDCl}_{3}\right) 7.23-7.47(\mathrm{~m}, 5 \mathrm{H}) ; 5.17$ $(\mathrm{dt}, J=8.8,3.2,1 \mathrm{H}) ; 3.65-3.76(\mathrm{ABX}, 2 \mathrm{H}) ; 3.49(\mathrm{~d}, J=2.9,1 \mathrm{H})$; $2.86-2.96(\mathrm{ABX}, 2 \mathrm{H}) ; 2.78(\mathrm{~m}, 1 \mathrm{H}) ; 1.03(\mathrm{~d}, J=6.9,3 \mathrm{H}) ; 0.87(\mathrm{~s}$, 9H); $0.04(\mathrm{~d}, J=2,6 \mathrm{H}),{ }^{13} \mathrm{C}$ NMR: $\left(125 \mathrm{MHz}, \mathrm{CDCl}_{3}\right) 214.67$; $142.85,128.47,127.52,125.60 ; 69.68 ; 65.56 ; 51.40 ; 48.98 ; 25.78$; 18.16; 12.59; -5.59, IR: (neat) 1708 (m), MS: (FI) $322\left(1.7, \mathrm{M}^{+}\right)$, $[\alpha]_{\mathrm{D}}{ }^{24}+69.11^{\circ}\left(\mathrm{CHCl}_{3}, \mathrm{c}=1.0\right)$; TLC: $R_{f}=0.19$ (hexane/EtOAc, $5 /$ 1, $\left.\mathrm{SiO}_{2}\right)$, SFC (1.4\% MeOH): $t_{R}(2 S, 5 R)-$ syn-7a $4.74 \min (95 \%)$; $(2 \mathrm{~S}, 5 S)$-anti-7a $5.21 \mathrm{~min}(5 \%)$, Anal. Calc for $\mathrm{C}_{18} \mathrm{H}_{30} \mathrm{O}_{3} \mathrm{Si}$ (322.51), Calc. C 67.03\%; H 9.38\%, Found: C 66.94\%; H 9.42\%

(2S,5S)-1-tert-Butyldimethylsilyloxy-2-methyl-5-hydroxy-5phenyl-3-pentanone (anti-7a)

${ }^{1} \mathrm{H}$ NMR: $\left(500 \mathrm{MHz}, \mathrm{CDCl}_{3}\right) 7.27-7.38(\mathrm{~m}, 5 \mathrm{H}) ; 5.14(\mathrm{dt}, J=9.6$, $2.8,1 \mathrm{H}) ; 3.67-3.77(\mathrm{ABX}, 2 \mathrm{H}) ; 3.50(\mathrm{~d}, J=3.0,1 \mathrm{H}) ; 2.84-2.97$ (ABX, 2H); $2.78(\mathrm{~m}, 1 \mathrm{H}) ; 1.05(\mathrm{~d}, J=7.1,3 \mathrm{H}) ; 0.88(\mathrm{~s}, 9 \mathrm{H}) ; 0.05$ $(\mathrm{d}, J=2,6 \mathrm{H}), \mathrm{SFC}(1.4 \% \mathrm{MeOH}):_{R}(2 S, 5 R)-s y n-7 \mathbf{a} 4.81 \mathrm{~min}$ (12\%); $(2 S, 5 S)$-anti-7a 5.16 min $(88 \%)$, Anal. Calc for $\mathrm{C}_{18} \mathrm{H}_{30} \mathrm{O}_{3} \mathrm{Si}$ (322.51), Calc. C 67.03\%; H 9.38\%, Found: C 66.81\%; H 9.20\%

(2S,5R)-1-tert-Butyldimethylsilyloxy-2-methyl-5-hydroxy-5-(1naphthyl)-3-pentanone (syn-7b)

${ }^{1} \mathrm{H}$ NMR: (500 MHz, $\left.\mathrm{CDCl}_{3}\right) 7.47$ - $8.02(\mathrm{~m}, 7 \mathrm{H}) ; 5.97$ (dt, $J=9.5$, $2.5,1 \mathrm{H}) ; 3.68-3.80(\mathrm{ABX}, 2 \mathrm{H}) ; 3.58(\mathrm{~d}, J=2.9,1 \mathrm{H}) ; 2.98-3.13$ $(\mathrm{ABX}, 2 \mathrm{H}) ; 2.79(\mathrm{~m}, 1 \mathrm{H}) ; 1.06(\mathrm{~d}, J=7.1,3 \mathrm{H}) ; 0.86(\mathrm{~s}, 9 \mathrm{H}) ; 0.04$ $(\mathrm{d}, J=3.9,6 \mathrm{H}), \mathrm{SFC}(12.5 \% \mathrm{MeOH}): t_{R}(2 S, 5 S)$-anti-7b $3.23 \mathrm{~min}$ (6\%); (2S, 5R)-syn-7b 4.12 min (94\%), Anal. Calc for $\mathrm{C}_{22} \mathrm{H}_{32} \mathrm{O}_{3} \mathrm{Si}$ (372.57), Calc. C 70.92\%; H 8.66\%, Found: C 70.74\%; H 8.60\%

(2S,5S)-1-tert-Butyldimethylsilyloxy-2-methyl-5-hydroxy-5-(1naphthyl)-3-pentanone (7b)

${ }^{1} \mathrm{H}$ NMR: (500 MHz, CDCl $\left.{ }_{3}\right) 7.47-8.02(\mathrm{~m}, 7 \mathrm{H}) ; 5.98(\mathrm{~m}, J=9.5$, $1 \mathrm{H}) ; 3.69-3.79(\mathrm{ABX}, 2 \mathrm{H}) ; 3.68(\mathrm{~d}, J=2.8,1 \mathrm{H}) ; 3.01-3.09$ (ABX, 2H); $2.82(\mathrm{~m}, 1 \mathrm{H}) ; 1.07$ (d, $J=7.1,3 \mathrm{H}) ; 0.88$ (s, 9H); 0.05 $\left(\mathrm{d}, J=3.9,6 \mathrm{H}, \mathrm{SiCH}_{3}\right), \mathrm{SFC}(12.5 \% \mathrm{MeOH}): t_{R}(2 S, 5 S)$-anti-7b 3.15 min $(89 \%)$; $(2 S, 5 R)-s y n-7 \mathbf{b} 4.11$ min $(11 \%)$, Anal. Calc for $\mathrm{C}_{22} \mathrm{H}_{32} \mathrm{O}_{3} \mathrm{Si}$ (372.57), Calc. C 70.92\%; H 8.66\%, Found: C 70.63\%; $\mathrm{H} 8.58 \%$

(2S,5R,6E)-1-tert-Butyldimethylsilyloxy-2-methyl-5-hydroxy-7phenyl-6-hepten-3-one (syn-7c)

${ }^{1} \mathrm{H}$ NMR: $\left(500 \mathrm{MHz}, \mathrm{CDCl}_{3}\right) 7.37(\mathrm{~d}, J=8.6,1 \mathrm{H}) ; 7.37(\mathrm{~d}, J=5.6$, $1 \mathrm{H}) ; 7.30(\mathrm{t}, J=7.7,2 \mathrm{H}) ; 7.23(\mathrm{t}, J=7.2,1 \mathrm{H}) ; 6.65(\mathrm{~d}, J=16.2$, $1 \mathrm{H}) ; 6.21(\mathrm{dd}, J=16.0,6.0,1 \mathrm{H}) ; 4.77(\mathrm{~m}, 1 \mathrm{H}) ; 3.66-3.77(\mathrm{ABX}$, $2 \mathrm{H}) ; 3.31(\mathrm{~d}, J=3.4,1 \mathrm{H}) ; 2.75-2.90(\mathrm{ABX}, 2 \mathrm{H}) ; 2.82(\mathrm{~m}, 1 \mathrm{H})$; $1.06(\mathrm{~d}, J=7.1,3 \mathrm{H}) ; 0.88(\mathrm{~s}, 9 \mathrm{H}) ; 0.05(\mathrm{~d}, J=5.7,6 \mathrm{H}), \mathrm{SFC}(7.5 \%$ $\mathrm{MeOH}): t_{R}(2 S, 5 R)-s y n-7 \mathbf{c} 2.74 \min (89 \%) ;(2 S, 5 S)$-anti-7c 3.26 $\min (11 \%)$, Anal. Calc for $\mathrm{C}_{20} \mathrm{H}_{32} \mathrm{O}_{3} \mathrm{Si}$ (348.55), Calc. C 68.92\%; H 9.38\%, Found: C $68.59 \%$; $\mathrm{H} 9.02 \%$

(2S,5S,6E)-1-tert-Butyldimethylsilyloxy-2-methyl-5-hydroxy-7phenyl-6-hepten-3-one (anti-7c)

${ }^{1} \mathrm{H}$ NMR: $\left(500 \mathrm{MHz}, \mathrm{CDCl}_{3}\right) 7.37(\mathrm{~d}, J=8.6,1.0,1 \mathrm{H}) ; 7.37$ (d, $J=7.7,1.0,1 \mathrm{H}) ; 7.31(\mathrm{t}, J=7.7,2 \mathrm{H}) ; 7.23(\mathrm{t}, J=7.2,1 \mathrm{H}) ; 6.67$ $(\mathrm{dd}, J=16.1,1.4,1 \mathrm{H}) ; 6.22(\mathrm{dd}, J=15.9,6.0,1 \mathrm{H}) ; 4.75(\mathrm{~m}, 1 \mathrm{H})$; 3.67 - 3.78 (ABX, 2H); $3.34(\mathrm{~d}, J=3.4,1 \mathrm{H}) ; 2.77-2.92(\mathrm{ABX}$, $2 \mathrm{H}) ; 2.84(\mathrm{~m}, 1 \mathrm{H}) ; 1.07(\mathrm{~d}, J=7.1,3 \mathrm{H}) ; 0.88(\mathrm{~s}, 9 \mathrm{H}) ; 0.05(\mathrm{~d}$, $J=3.7,6 \mathrm{H}), \operatorname{SFC}(7.5 \% \mathrm{MeOH}): t_{R}(2 S, 5 R)-$ syn-7c $2.55 \mathrm{~min}$ (19\%); $(2 S, 5 S)$-anti-7c $2.96 \min (81 \%)$, Anal. Calc for $\mathrm{C}_{20} \mathrm{H}_{32} \mathrm{O}_{3} \mathrm{Si}$ (348.55), Calc. C 68.92\%; H 9.38\%, Found: C 68.56\%; H 9.14\%

(2S,5R,6E)-1-tert-Butyldimethylsilyloxy-2,6-dimethyl-5-hydroxy-7-phenyl-6-hepten-3-one (syn-7d)

${ }^{1} \mathrm{H}$ NMR: $\left(500 \mathrm{MHz}, \mathrm{CDCl}_{3}\right) 7.33$ (t, $\left.J=7.7,2 \mathrm{H}\right) ; 7.27$ (d, $J=5.4$, $2 \mathrm{H}) ; 7.21(\mathrm{t}, J=7.4,1 \mathrm{H}) ; 6.58(\mathrm{~s}, 1 \mathrm{H}) ; 4.64(\mathrm{dt}, J=9.5,2.61 \mathrm{H})$; 3.67 - $3.77(\mathrm{ABX}, 2 \mathrm{H}) ; 3.27(\mathrm{~d}, J=3.0,1 \mathrm{H}) ; 2.77-2.89(\mathrm{~m}, 3 \mathrm{H})$; $1.88(\mathrm{~s}, 3 \mathrm{H}) ; 1.06(\mathrm{~d}, J=7.1,3 \mathrm{H}) ; 0.88(\mathrm{~s}, 9 \mathrm{H}) ; 0.06(\mathrm{~d}, J=5.1$, $6 \mathrm{H}), \mathrm{SFC}(7.5 \% \mathrm{MeOH}): t_{R}(2 S, 5 R)-s y n-7 \mathbf{d} 2.76 \mathrm{~min}(91 \%) ;(2 S$, 5S)-anti-7d $3.03 \mathrm{~min}(9 \%)$, Anal. Calc for $\mathrm{C}_{21} \mathrm{H}_{34} \mathrm{O}_{3} \mathrm{Si}(362.58)$, Calc. C $69.56 \%$; H 9.45\%, Found: C $69.53 \%$; H $9.45 \%$

(2S,5S,6E)-1-tert-Butyldimethylsilyloxy-2,6-dimethyl-5-hydroxy-7-phenyl-6-hepten-3-one (anti-7d)

${ }^{1} \mathrm{H}$ NMR: $\left(500 \mathrm{MHz}, \mathrm{CDCl}_{3}\right) 7.33(\mathrm{t}, J=7.6,2 \mathrm{H}) ; 7.27(\mathrm{~d}, J=6.6$, $2 \mathrm{H}) ; 7.21(\mathrm{t}, J=7.2,1 \mathrm{H}) ; 6.58(\mathrm{~s}, 1 \mathrm{H}) ; 4.62(\mathrm{dt}, J=9.0,2.81 \mathrm{H})$; 3.68 - $3.78(\mathrm{ABX}, 2 \mathrm{H}) ; 3.30(\mathrm{~d}, J=2.9,1 \mathrm{H}) ; 2.78-2.88(\mathrm{~m}, 3 \mathrm{H})$; $1.88(\mathrm{~d}, J=1.3,3 \mathrm{H}) ; 1.08(\mathrm{~d}, J=7.1,3 \mathrm{H}) ; 0.89(\mathrm{~s}, 9 \mathrm{H}) ; 0.06(\mathrm{~d}$, $J=5.1,6 \mathrm{H})$, SFC $(7.5 \% \mathrm{MeOH}): t_{R}(2 S, 5 R)-s y n-7 \mathbf{d} 2.65 \mathrm{~min}$ (13\%); $(2 S, 5 S)$-anti-7d $2.91 \mathrm{~min}(87 \%)$, Anal. Calc for $\mathrm{C}_{21} \mathrm{H}_{34} \mathrm{O}_{3} \mathrm{Si}$ (362.58), Calc. C 69.56\%; H 9.45\%, Found: C 69.52\%; H 9.49\%

(2S,5R,6E)-1-tert-Butyldimethylsilyloxy-2-methyl-5-hydroxy-6octen-3-one (syn-7e)

${ }^{1} \mathrm{H}$ NMR: $\left(500 \mathrm{MHz}, \mathrm{CDCl}_{3}\right)$; $5.71(\mathrm{dqd}, J=15.4,6.6,1.0,1 \mathrm{H})$; 5.49 (ddq, $J=15.5,6.6,1.4,1 \mathrm{H}) ; 4.52(\mathrm{~m}, 1 \mathrm{H}) ; 3.63$ - 3.74 (ABX, $2 \mathrm{H}) ; 3.13(\mathrm{~d}, J=3.4,1 \mathrm{H}) ; 2.64-2.78(\mathrm{ABX}, 2 \mathrm{H}) ; 2.78(\mathrm{~m}, 1 \mathrm{H})$; $1.69(\mathrm{dt}, J=6.6,0.8,3 \mathrm{H}) ; 1.02(\mathrm{~d}, J=6.8,3 \mathrm{H}) ; 0.87(\mathrm{~s}, 9 \mathrm{H}) ; 0.03$ $(\mathrm{d}, J=6.4,6 \mathrm{H}), \mathrm{SFC}(5$-benzoyloxy-7e, $1.5 \% \mathrm{MeOH}): t_{R}(2 S, 5 R)$ syn-5-benzoyloxy-7e 4.44 min (83\%); (2S, 5S)-anti-5-benzoyloxy7e 5.26 min (17\%), Anal. Calc for $\mathrm{C}_{15} \mathrm{H}_{20} \mathrm{O}_{3} \mathrm{Si}$ (286.48), Calc. C $62.89 \%$; H $10.55 \%$, Found: C 62.69\%; H $10.67 \%$

(2S,5S,6E)-1-tert-Butyldimethylsilyloxy-2-methyl-5-hydroxy-6octen-3-one (anti-7e)

${ }^{1} \mathrm{H}$ NMR: $\left(500 \mathrm{MHz}, \mathrm{CDCl}_{3}\right) ; 5.69(\mathrm{dqd}, J=15.1,6.6,1.0,1 \mathrm{H})$; 5.48 (ddq, $J=15.5,6.6,1.2,1 \mathrm{H}) ; 4.47(\mathrm{~m}, 1 \mathrm{H}) ; 3.61-3.74(\mathrm{ABX}$, $2 \mathrm{H}) ; 3.15(\mathrm{~d}, J=3.4,1 \mathrm{H}) ; 2.62-2.77(\mathrm{~m}, 3 \mathrm{H}) ; 1.67(\mathrm{dd}, J=6.3$, 
$0.7,3 \mathrm{H}) ; 1.02(\mathrm{dd}, J=6.9,1.0,3 \mathrm{H}) ; 0.85(\mathrm{~d}, J=0.9,9 \mathrm{H}) ; 0.02(\mathrm{~d}$, $J=3.2,6 \mathrm{H}), \mathrm{SFC}$ (5-benzoyloxy-7e, $1.5 \% \mathrm{MeOH}): t_{R}(2 S, 5 R)$-syn5-benzoyloxy-7e $4.47 \mathrm{~min}$ (26\%); (2S, 5S)-anti-5-benzoyloxy-7e 5.14 min (74\%), Anal. Calc for $\mathrm{C}_{15} \mathrm{H}_{20} \mathrm{O}_{3} \mathrm{Si}$ (286.48), Calc. C $62.89 \%$; H $10.55 \%$, Found: C 62.70\%; H $10.57 \%$

(2S,5R)-1-tert-Butyldimethylsilyloxy-2,6-dimethyl-5-hydroxy6-hepten-3-one (syn-7f)

${ }^{1} \mathrm{H}$ NMR: $\left(500 \mathrm{MHz}, \mathrm{CDCl}_{3}\right) ; 5.01(\mathrm{~s}, 1 \mathrm{H}) ; 4.86(\mathrm{~s}, 1 \mathrm{H}) ; 4.51(\mathrm{~d}$, $J=9.5,1 \mathrm{H}) ; 3.65-3.74(\mathrm{ABX}, 2 \mathrm{H}) ; 3.17(\mathrm{~d}, J=3.2,1 \mathrm{H}) ; 2.80(\mathrm{~m}$, $1 \mathrm{H}) ; 2.67-2.82(\mathrm{ABX}, 2 \mathrm{H}) ; 1.75(\mathrm{~s}, 3 \mathrm{H}) ; 1.03(\mathrm{~d}, J=6.8,3 \mathrm{H})$; $0.87(\mathrm{~s}, 9 \mathrm{H}) ; 0.04(\mathrm{~d}, J=5.8,6 \mathrm{H})$, SFC (5-benzoyloxy-7f, $1.5 \%$ $\mathrm{MeOH}): t_{R}(2 S, 5 S)$-anti-5-benzoyloxy-7f $5.26 \mathrm{~min}(6 \%) ;(2 S, 5 R)$ syn-5-benzoyloxy-7f 4.44 min (94\%), Anal. Calc for $\mathrm{C}_{15} \mathrm{H}_{20} \mathrm{O}_{3} \mathrm{Si}$ (286.48), Calc. C $62.89 \%$; H $10.55 \%$, Found: C $62.81 \%$; H $10.68 \%$

(2S,5S)-1-tert-Butyldimethylsilyloxy-2,6-dimethyl-5-hydroxy6-hepten-3-one (anti-7f)

${ }^{1} \mathrm{H}$ NMR: $\left(500 \mathrm{MHz}, \mathrm{CDCl}_{3}\right) ; 5.01(\mathrm{~m}, 1 \mathrm{H}) ; 4.86(\mathrm{t}, J=1.2,1 \mathrm{H})$; $4.49(\mathrm{~m}, 1 \mathrm{H}) ; 3.65-3.76(\mathrm{ABX}, 2 \mathrm{H}) ; 3.21(\mathrm{~d}, J=3.2,1 \mathrm{H}) ; 2.80(\mathrm{~m}$, $1 \mathrm{H}) ; 2.67-2.75(\mathrm{ABX}, 2 \mathrm{H}) ; 1.75(\mathrm{~s}, 3 \mathrm{H}) ; 1.05(\mathrm{~d}, J=6.9,3 \mathrm{H})$; $0.87(\mathrm{~s}, 9 \mathrm{H}) ; 0.04(\mathrm{~d}, J=3.6,6 \mathrm{H})$, SFC (5-benzoyloxy-7f, $1.5 \%$ $\mathrm{MeOH}): t_{R}(2 S, 5 S)$-anti-5-benzoyloxy-7f $5.26 \mathrm{~min}(89 \%)$; $(2 S$, 5R)-syn-5-benzoyloxy-7f $4.44 \mathrm{~min}(11 \%)$, Anal. Calc for $\mathrm{C}_{15} \mathrm{H}_{20} \mathrm{O}_{3} \mathrm{Si}$ (286.48), Calc. C $62.89 \%$; H $10.55 \%$, Found: C $62.50 \%$; H $10.51 \%$

(2S,5R,6E)-1-tert-Butyldimethylsilyloxy-2,6-dimethyl-5-hydroxy-6-octen-3-one (syn-7g)

$\left(500 \mathrm{MHz}, \mathrm{CDCl}_{3}\right) ; 5.53$ (qt, $\left.J=6.5,1.2,1 \mathrm{H}\right) ; 4.46$ (td, $J=6.4,1.5$, $1 \mathrm{H}) ; 3.64-3.74(\mathrm{ABX}, 2 \mathrm{H}) ; 3.06(\mathrm{~d}, J=2.7,1 \mathrm{H}) ; 2.79(\mathrm{~m}, 1 \mathrm{H})$ $2.64-2.76(\mathrm{ABX}, 2 \mathrm{H}) ; 1.63(\mathrm{~d}, J=1.0,3 \mathrm{H}) ; 1.61(\mathrm{~d}, J=6.6,3 \mathrm{H})$; $1.03(\mathrm{~d}, J=7.1,3 \mathrm{H}) ; 0.87(\mathrm{~s}, 9 \mathrm{H}) ; 0.03(\mathrm{~d}, J=6.9,6 \mathrm{H}), \mathrm{SFC}(5-$ benzoyloxy-7g, $2.5 \% \mathrm{MeOH}): t_{R}(2 S, 5 R)$-syn-5-benzoyloxy-7g $2.94 \mathrm{~min}(96 \%)$; (2S, 5S)-anti-5-benzoyloxy-7g $3.45 \mathrm{~min}(4 \%)$, Anal. Calc for $\mathrm{C}_{15} \mathrm{H}_{20} \mathrm{O}_{3} \mathrm{Si}(300.51)$, Calc. C 63.95\%; H $10.73 \%$, Found: C $64.04 \%$; H $10.86 \%$

(2S,5S,6E)-1-tert-Butyldimethylsilyloxy-2,6-dimethyl-5-hydroxy-6-octen-3-one (anti-7g)

$\left(500 \mathrm{MHz}, \mathrm{CDCl}_{3}\right) ; 5.54(\mathrm{qd}, J=6.7,1.0,1 \mathrm{H}) ; 4.44(\mathrm{~d}, J=9.8$, $1 \mathrm{H}) ; 3.64$ - $3.76(\mathrm{ABX}, 2 \mathrm{H}) ; 3.11(\mathrm{~d}, J=2.7,1 \mathrm{H}) ; 2.79(\mathrm{~m}, 1 \mathrm{H})$; $2.64-2.80(\mathrm{ABX}, 2 \mathrm{H}) ; 1.63(\mathrm{~d}, J=1.0,3 \mathrm{H}) ; 1.61(\mathrm{~d}, J=6.8,3 \mathrm{H})$; $1.05(\mathrm{~d}, J=7.0,3 \mathrm{H}) ; 0.88(\mathrm{~s}, 9) ; 0.04(\mathrm{~d}, J=3.7,6 \mathrm{H}), \mathrm{SFC}$ (5-benzoyloxy-7g, $2.5 \% \mathrm{MeOH}): t_{R}(2 S, 5 R)$-syn-5-benzoyloxy-7g 3.03 $\min (18 \%)$; (2S, 5S)-anti-5-benzoyloxy-7g $3.48 \mathrm{~min}(82 \%)$, Anal. Calc for $\mathrm{C}_{15} \mathrm{H}_{20} \mathrm{O}_{3} \mathrm{Si}$ (300.51), Calc. C 63.95\%; H 10.73\%, Found: C $64.75 \%$; H $10.87 \%$

(2S,5R)-1-tert-Butyldimethylsilyloxy-2-methyl-5-hydroxy-7phenyl-6-heptyn-3-one (syn-7h)

${ }^{1} \mathrm{H}$ NMR: $\left(500 \mathrm{MHz}, \mathrm{CDCl}_{3}\right) 7.28-7.43(\mathrm{~m}, 5 \mathrm{H}) ; 5.01-5.06(\mathrm{~m}$, $1 \mathrm{H}) ; 3.67-3.77(\mathrm{ABX}, 2 \mathrm{H}) ; 3.33(\mathrm{~d}, J=5.1,1 \mathrm{H}) ; 2.96-3.13$ $(\mathrm{ABX}, 2 \mathrm{H}) ; 2.82(\mathrm{~m}, 1 \mathrm{H}) ; 1.08(\mathrm{~d}, J=7.1,3 \mathrm{H}) ; 0.88(\mathrm{~s}, 9 \mathrm{H}) ; 0.06$ $(\mathrm{d}, J=5.1,6 \mathrm{H}), \mathrm{SFC}(7.5 \% \mathrm{MeOH}): t_{R}(2 S, 5 R)-\operatorname{syn}-\mathbf{7 h} 3.35 \mathrm{~min}$ (76\%); $(2 \mathrm{~S}, 5 \mathrm{~S})$-anti-7h $3.82 \mathrm{~min}(24 \%)$, Anal. Calc for $\mathrm{C}_{20} \mathrm{H}_{30} \mathrm{O}_{3} \mathrm{Si}$ (346.54), Calc. C 69.32\%; H 8.73\%, Found: C 69.20\%; H 8.77\%

(2S,5S)-1-tert-Butyldimethylsilyloxy-2-methyl-5-hydroxy-7phenyl-6-heptyn-3-one (anti-7h)

${ }^{1} \mathrm{H}$ NMR: $\left(500 \mathrm{MHz}, \mathrm{CDCl}_{3}\right) 7.28-7.43(\mathrm{~m}, 5 \mathrm{H}) ; 5.01-5.06(\mathrm{~m}$, $1 \mathrm{H}) ; 3.67-3.79(\mathrm{ABX}, 2 \mathrm{H}) ; 3.29(\mathrm{~d}, J=5.1,1 \mathrm{H}) ; 2.97-3.13$ $(\mathrm{ABX}, 2 \mathrm{H}) ; 2.82(\mathrm{~m}, 1 \mathrm{H}) ; 1.09(\mathrm{~d}, J=7.1,3 \mathrm{H}) ; 0.88(\mathrm{~s}, 9 \mathrm{H}) ; 0.05$ $(\mathrm{d}, J=3.9,6 \mathrm{H}), \mathrm{SFC}(7.5 \% \mathrm{MeOH}): t_{R}(2 S, 5 R)-s y n-7 \mathbf{h} 3.47 \mathrm{~min}$ (43\%); (2S, 5S)-anti-7h $3.94 \min (57 \%)$, Anal. Calc for $\mathrm{C}_{20} \mathrm{H}_{30} \mathrm{O}_{3} \mathrm{Si}$ (346.54), Calc. C 69.32\%; H 8.73\%, Found: C 69.13\%; H 8.75\%

(2S,5R)-1-tert-Butyldimethylsilyloxy-2-methyl-5-hydroxy-7phenyl-3-heptanone (syn-7i)

${ }^{1} \mathrm{H}$ NMR: $\left(500 \mathrm{MHz}, \mathrm{CDCl}_{3}\right) 7.17-7.30(\mathrm{~m}, 5 \mathrm{H}) ; 4.07(\mathrm{~m}, 1 \mathrm{H})$; $3.62-3.73(\mathrm{ABX}, 2 \mathrm{H}) ; 3.24(\mathrm{dd}, J=3.5,0.8,1 \mathrm{H}) ; 2.56-2.85(\mathrm{~m}$, $5 \mathrm{H}) ; 1.65-1.86(2 \mathrm{~m}, 2 \mathrm{H}) ; 1.02(\mathrm{~d}, J=7.0,3 \mathrm{H}) ; 0.86(\mathrm{~s}, 9 \mathrm{H}) ; 0.03$ $(\mathrm{d}, J=9.0,6 \mathrm{H}), \mathrm{SFC}(5 \% \mathrm{MeOH}): t_{R}(2 S, 5 R)-$ syn-7i $3.45 \mathrm{~min}$ (91\%); (2S,5S)-anti-7i 3.87 min (9\%), Anal. Calc for $\mathrm{C}_{20} \mathrm{H}_{34} \mathrm{O}_{3} \mathrm{Si}$ (350.57), Calc. C 68.52\%; H 9.78\%, Found: C 68.59\%; H 9.91\%

(2S,5S)-1-tert-Butyldimethylsilyloxy-2-methyl-5-hydroxy-7phenyl-3-heptanone (anti-7i)

${ }^{1} \mathrm{H}$ NMR: $\left(500 \mathrm{MHz}, \mathrm{CDCl}_{3}\right) 7.17-7.30(\mathrm{~m}, 5 \mathrm{H}) ; 4.03(\mathrm{~m}, 1 \mathrm{H})$; 3.62 - $3.75(\mathrm{ABX}, 2 \mathrm{H}) ; 3.28(\mathrm{dd}, J=3.2,0.7,1 \mathrm{H}) ; 2.56-2.85(\mathrm{~m}$, $5 \mathrm{H}) ; 1.66-1.87(2 \mathrm{~m}, 2 \mathrm{H}) ; 1.03(\mathrm{~d}, J=7.0,3 \mathrm{H}) ; 0.87(\mathrm{~s}, 9 \mathrm{H}) ; 0.04$ $(\mathrm{d}, J=3.2,6 \mathrm{H})$, SFC $(5 \% \mathrm{MeOH}): t_{R}(2 S, 5 R)-s y n-7 \mathbf{i} 3.51 \mathrm{~min}$ (29\%); $(2 S, 5 S)$-anti-7i $3.88 \mathrm{~min}(71 \%)$, Anal. Calc for $\mathrm{C}_{20} \mathrm{H}_{34} \mathrm{O}_{3} \mathrm{Si}$ (350.57), Calc. C 68.52\%; H 9.78\%, Found: C 68.64\%; H 9.78\%

(2S,5R)-1-tert-Butyldimethylsilyloxy-2-methyl-5-hydroxy-5-cyclohexyl-3-pentanone (syn-7j)

${ }^{1} \mathrm{H}$ NMR: (500 MHz, $\left.\mathrm{CDCl}_{3}\right) 3.83(\mathrm{~m}, 1 \mathrm{H}) ; 3.64$ - $3.76(\mathrm{ABX}, 2 \mathrm{H})$; $3.12(\mathrm{~d}, J=3.4,1 \mathrm{H}) ; 2.82(\mathrm{~m}, 1 \mathrm{H}) ; 2.54-2.77(\mathrm{ABX}, 2 \mathrm{H}) ; 1.66-$ $1.89(5 \mathrm{H}) ; 1.00-1.38(6 \mathrm{H}) ; 1.03(\mathrm{~d}, J=7.1,3 \mathrm{H}) ; 0.88(\mathrm{~s}, 9 \mathrm{H}) ; 0.05$ $(\mathrm{d}, J=5.6,6 \mathrm{H})$, SFC (5-benzoyloxy-7j, $2 \% \mathrm{MeOH}): t_{R}(2 S, 5 R)-$ syn-5-benzoyloxy-7j 4.59 min (94\%); $(2 S, 5 S)$-anti-5-benzoyloxy$7 j 5.52$ min (6\%), Anal. Calc for $\mathrm{C}_{18} \mathrm{H}_{36} \mathrm{O}_{3} \mathrm{Si}$ (328.56), Calc. C $65.80 \%$; H $11.04 \%$, Found: C $65.92 \%$; H $11.26 \%$

(2S,5S)-1-tert-Butyldimethylsilyloxy-2-methyl-5-hydroxy-5-cyclohexyl-3-pentanone (anti-7j)

${ }^{1} \mathrm{H}$ NMR: (500 MHz, $\left.\mathrm{CDCl}_{3}\right) 3.81(\mathrm{~m}, 1 \mathrm{H}) ; 3.64$ - $3.78(\mathrm{ABX}, 2 \mathrm{H})$; $3.15(\mathrm{~d}, J=3.5,1 \mathrm{H}) ; 2.81(\mathrm{~m}, 1 \mathrm{H}) ; 2.54-2.73(\mathrm{ABX}, 2 \mathrm{H}) ; 1.66-$ $1.89(5 \mathrm{H}) ; 0.97-1.42(6 \mathrm{H}) ; 1.05(\mathrm{~d}, J=7.0,3 \mathrm{H}) ; 0.89(\mathrm{~s}, 9 \mathrm{H}) ; 0.06$ $(\mathrm{d}, J=2.7,6 \mathrm{H})$, SFC (5-benzoyloxy-7j, $2 \% \mathrm{MeOH}): t_{R}(2 S, 5 R)-$ syn-5-benzoyloxy-7j $4.41 \mathrm{~min}(20 \%)$; $(2 S, 5 S)$-anti-5-benzoyloxy$7 j 5.17$ min (80\%), Anal. Calc for $\mathrm{C}_{18} \mathrm{H}_{36} \mathrm{O}_{3} \mathrm{Si}$ (328.56), Calc. C $65.80 \%$; H $11.04 \%$, Found: C $65.80 \%$; H $11.19 \%$

(2S,5R)-1-tert-Butyldimethylsilyloxy-2,6,6-trimethyl-5-hydroxy-3-heptanone (syn-7k)

${ }^{1}$ H NMR: (500 MHz, benzene- $\left.d_{6}\right) 3.84(\mathrm{dt}, J=10.5,2.2,1 \mathrm{H}) ; 3.41$ - 3.64 (ABX, 2H); 3.17 (d, $J=2.9,1 \mathrm{H}$ ); $2.37-2.59$ (ABX, 2H); $2.48(\mathrm{~m}, 1 \mathrm{H}) ; 0.95(\mathrm{~s}, 9 \mathrm{H}) ; 0.91(\mathrm{~s}, 9 \mathrm{H}) ; 0.82(\mathrm{~d}, J=6.8,3 \mathrm{H}) ;-0.01$ $(\mathrm{d}, J=1.9,6 \mathrm{H})$, Anal. Calc for $\mathrm{C}_{16} \mathrm{H}_{34} \mathrm{O}_{3} \mathrm{Si}$ (302.52), Calc. C 63.52\%; H 11.33\%, Found: C 63.41\%; H $11.44 \%$

(2S,5S)-1-tert-Butyldimethylsilyloxy-2,6,6-trimethyl-5-hydroxy-3-heptanone (anti-7k)

${ }^{1} \mathrm{H}$ NMR: (500 MHz, benzene- $\left.d_{6}\right) 3.77$ (dt, $\left.J=9.2,3.2,1 \mathrm{H}\right) ; 3.44$ $3.64(\mathrm{ABX}, 2 \mathrm{H}) ; 3.24(\mathrm{~d}, J=3.0,1 \mathrm{H}) ; 2.37-2.59(\mathrm{~m}, 3 \mathrm{H}) ; 0.94(\mathrm{~s}$, $9 \mathrm{H}) ; 0.93(\mathrm{~s}, 9 \mathrm{H}) ; 0.85(\mathrm{~d}, J=6.8,3 \mathrm{H}) ; 0.01(\mathrm{~s}, 6 \mathrm{H})$, Anal. Calc for $\mathrm{C}_{16} \mathrm{H}_{34} \mathrm{O}_{3} \mathrm{Si}$ (302.52), Calc. C 63.52\%; $\mathrm{H} 11.33 \%$, Found: $\mathrm{C}$ $63.27 \%$; $\mathrm{H} 11.43 \%$

\section{Acknowledgement}

We are grateful to the National Science Foundation (NSF CHE9803124) for generous financial support. S.F. thanks the Abbott Laboratories for a Graduate Fellowship in Synthetic Organic Chemistry. We also thank Dr. Alan Walker (Kaneka America) for a gift of (+)-methyl $\beta$-hydroxyisobutyrate.

\section{References and Notes}

(1) Reviews: (a) Paterson, I.; Cowden, C. J.; Wallace, D. J. In Modern Carbonyl Chemistry, Otera, J., Ed.; Wiley-VCH: Weinheim, 2000; Chapt. 9. (b) Cowden, C. J.; Paterson, I. Org. React. 1997, 51, 1.

(2) For aldol reactions of $\alpha$-unsubstituted enolates, see: (a) Braun, M. Angew. Chem., Int. Ed. Engl. 1987, 26, 24-37. (b) Braun, M. In Stereoselective Synthesis, Methods of Organic Chemistry (Houben-Weyl); Edition E21; Helmchen, G.; Hoffman, R.; Mulzer, J.; Schaumann, E. Eds.; Thieme: Stuttgart, 1996; Vol. 3; pp 1622-1624. (c) Corey, E. J.; Cywin, C. L.; Roper, T. D. Tetrahedron Lett. 1992, 33, 6907. 
(d) Ishihara, K.; Maruyama, T.; Mouri, M.; Gao, Q.; Furuta, K.; Yamamoto, H. Bull. Chem. Soc. Jpn. 1993, 66, 3483. (e) Mikami, K.; Matsukawa, S. J. Am. Chem. Soc. 1993, 115, 7039. (f) Carreira, E. M.; Lee, W.; Singer, R. A. J. Am. Chem. Soc. 1995, 117, 3649. (g) Sodeoka, M.; Tokunoh, R.;

Miyazaki, F.; Hagiwara, E.; Shibasaki, M. Synlett 1997, 463. (h) Ando, A.; Miura, T.; Tatematsu, T.; Shioiri, T. Tetrahedron Lett. 1993, 34, 1507. (i) Yamamoto, H.; Yanagisawa, A.; Matsumoto, Y.; Nakashima, H.; Asakawa, K. J. Am. Chem. Soc. 1997, 119, 9319. (j) Yamada, Y. M. A.; Yoshikawa, N.; Sasai, H.; Shibasaki, M. Angew. Chem., Int. Ed. Engl. 1997, 36, 1871

(3) For a general discussion of the aldol transition structures see: (a) Evans, D. A; Nelson, J. V.; Taber, T. R. Stereoselective Aldol Condensations. In Topics in Stereochemistry; Eliel, E. L., Wilen, S. H., Eds.; Wiley Interscience: New York, 1982; Vol. 13, 1-116. (b) Carreira, E. M. In Modern Carbonyl Chemistry, Otera, J., Ed.; Wiley-VCH: Weinheim, 2000; Chapt. 8. (c) Ref 2b pp 1603-1612. (d) Li, Y.; Paddon-Row, M. N.; Houk, K. N. J. Org. Chem. 1990, 55, 481.

(4) (a) Denmark, S. E.; Stavenger, R. A. Acc. Chem. Res. 2000, 33, 432. (b) Denmark, S. E.; Stavenger, R. A. J. Am. Chem. Soc. 2000, 122, 8837.
(5) (a) Heathcock, C. H.; White, C. T.; Morrison, J. J. VanDerveer, D. J. Org. Chem. 1981, 56, 1296. (b) Heathcock, C. H.; Pirrung, M. C.; Lampe, J.; Buse, C. T.; Young, S. D. J. Org. Chem. 1981, 56, 2290. (c) Martin, V. A.; Albizati, K. F. J. Org. Chem. 1988, 53, 5986.

(6) (a) Paterson, I.; Goodman, J. M.; Isaka, M. Tetrahedron Lett. 1989, 30, 7121. (b) Paterson, I.; Oballa, R. M. Tetrahedron Lett. 1997, 38, 8421.

(7) Luke, G. P.; Morris, J. J. Org. Chem. 1995, 60, 3013.

(8) Denmark, S. E.; Stavenger, R. A.; Winter, S. B. D.; Wong, K.T.; Barsanti, P. A. J. Org. Chem. 1998, 63, 9517-9523.

(9) Denmark. S. E.; Pham, S. M. Unpublished results.

(10) For a definition of these terms see: Denmark, S. E.; Almstead, N. G. In Modern Carbonyl Chemistry; Otera, J., Ed.; WileyVCH: Weinheim, 2000; Chapt. 10.

(11) Assignment of the major diastereomer in all cases is based on analogy to benzaldehyde.

(12) We believe that the slow rate seen for aliphatic aldehydes in general in these reactions does not arise from enolization, but rather from formation of chlorohydrin-type adducts. T. Wynn, S. K. Ghosh, unpublished results from these laboratories.

(13) All chiral stationary phase analytical chromatographic determinations were performed on a Berger Instruments Supercritical Fluid Chromatograph (SFC) operating at 150 bar and $3.0 \mathrm{~mL} / \mathrm{min}$ fitted with a Chiralpak OD column.

Article Identifier:

1437-2096,E;2001,0,SI,1024,1029,ftx,en;Y05001ST.pdf 